\title{
Sensoriamento Remoto na Análise de Variáveis Ambientais Influenciadas pela Implantação da Usina Hidrelétrica de Belo Monte (PA)
}

\section{Remote Sensing in the Analysis of Environmental Variables Influenced by the Implementation of the Belo Monte Hydroelectric Power Plant, PA, BRAZIL}

Albertino Monteiro Neto

Graduando em Engenharia ambiental e de Energias Renováveis,

Universidade Federal Rural da Amazônia, Brasil albertino.monteiro.neto@gmail.com

Lucas Mota Batista Mestrando no Programa de Pós-Graduação em Ciências Ambientais da Universidade Federal do Pará (UFPA), brasil lucasmotab4@gmail.com

Marina Costa de Sousa Mestranda no Programa de Pós-Graduação em Ciências Ambientais da Universidade Federal do Pará (UFPA), Brasil sousamarina.mcs@gmail.com

Kemuel Maciel Freitas Graduando em Engenharia ambiental e de Energias Renováveis, Universidade Federal Rural da Amazônia, Brasil kemuel.macielf@gmail.com

Suzana Romeiro Araújo Engenheira Agrônoma, Professora - Instituto Socioambiental e dos Recursos Hídricos, Universidade Federal Rural da Amazônia, Brasil suzanaromeiro@yahoo.com.br

\section{Resumo}

A Usina Hidrelétrica de Belo Monte (UHBM) favoreceu o crescimento urbano na região em seu entorno, gerando impactos ambientais de uso e ocupação do solo e microclima. Para melhor compreensão destes impactos, o uso de geotecnologias tem auxiliado no monitoramento de determinadas variáveis ambientais, como a Temperatura de Superfície Terrestre (TST) e índices espectrais, tais como o Normalized difference vegetation index (NDVI) e Normalized Difference Built-up Index (NDBI), adotadas neste estudo. Foram utilizadas imagens dos satélites LANDSAT 5 e LANDSAT 8, referentes aos anos de 2011 e 2020, para aferição da TST, NDVI e NDBI. Os resultados indicaram que após a consolidação do empreendimento, uma área de $96 \mathrm{~km}^{2}$ passou a ser alagada e $203 \mathrm{~km}^{2}$ de área vegetada foi convertida em ambiente construído. Houve aumento da TST no período analisado, com uma diferença de mais de $8^{\circ} \mathrm{C}$ entre as máximas, chegando a $34{ }^{\circ} \mathrm{C}$. Ademais, os valores dos índices espectrais foram submetidos à análise estatística, através de correlação linear de Pearson, em comparação com a variável de temperatura. Estatisticamente observaram-se relações de influência negativa $(-0,48)$ entre as variáveis TST e NDVI, e positiva $(0,69)$ entre TST e NDBI. De modo geral, a construção da UHBM gerou mudanças expressivas no espaço, com significativa alteração do microclima. A perda de parte da área vegetada e conversão em ambiente construído/alagado está relacionada com o aumento da TST.

Palavras-chave:Índices espectrais; Impactos ambientais; Geotecnologias. 


\begin{abstract}
The Belo Monte Hydroelectric Power Plant (BMHPP) has favored urban growth in the surrounding region, causing environmental impacts related to microclimate, land use and occupation, among others. The use of geotechnologies can help in the monitoring of certain environmental variables, such as Terrestrial Surface Temperature (TST), Normalized difference vegetation index (NDVI) and Normalized Difference Built-up Index (NDBI), adopted in this study. LANDSAT 5 and LANDSAT 8 satellite images, referring to the years 2011 and 2020, were used to calculate TST, NDVI and NDBI. The results indicate that an area of $96 \mathrm{~km}^{2}$ began to be flooded, in addition to 203 $\mathrm{km}^{2}$ of vegetated area converted into a built environment after the consolidation of the enterprise. TST increased in the analyzed period, with a difference of more than $8{ }^{\circ} \mathrm{C}$ between the maximums, reaching $34^{\circ} \mathrm{C}$. In addition, the values of the spectral indices were subjected to statistical analysis, through Pearson's linear correlation, in comparison with the temperature variable. Statistically, there was a negative influence relationship (-0.48) between the variables TST and NDVI and positive influence relationship (0.69) between TST and NDBI. In general, the construction of the BMHPP generated substantial changes in space, with significant change in the microclimate. The loss of part of the vegetated area and its conversion into a built/flooded environment is related to the increase in TST.
\end{abstract}

Keywords: Spectral indices; Environmental impacts; Geotechnologies.

\title{
1. INTRODUÇÃO
}

A construção de usinas hidrelétricas é parte do planejamento do Estado brasileiro como suporte à industrialização do país, buscando atender demandas por matéria prima (FERREIRA; SALATI, 2005). Durante a fase de implantação destes empreendimentos muitas transformações ambientais e socioeconômicas ocorrem e, por vezes, extrapolam a região onde estão localizados.

A alteração do microclima local, por exemplo, é um reflexo do impacto da ocupação e da atividade antrópica sobre o meio ambiente (NASCIMENTO; DE OLIVEIRA, 2011). Sabe-se que a intensificação do processo de urbanização provocada por grandes empreendimentos se estabelece por meio da mudança no uso do solo e modificações da cobertura natural do mesmo (FREITAS et al., 2012). Os impactos decorrentes do desenvolvimento de uma área urbana sobre os processos hidrológicos estão associados à forma de ocupação da terra, e esta, ao aumento das superfícies impermeáveis (BEZERRA et al. 2018).

A usina hidrelétrica de Belo Monte (UHEBM), construída no estado do Pará, é um exemplo de empreendimento que favoreceu o crescimento urbano na região, mas que também tem gerado expressivos impactos ambientais, desde a sua implantação (FREIRE et al., 2018; DE SOUZA et al., 2018). No entorno da região afetada, existem diferentes Unidades de Conservação (UC), como reservas indígenas, que foram diretamente impactadas pela construção da UHEBM principalmente as terras dos índios Juruna e dos Arara, de Volta Grande (ARAUJO et al., 2015). Os indígenas e ribeirinhos representam grupos sociais vulneráveis, sendo os principais atingidos por 
este mega projeto, e alertam a falta de participação no planejamento e na tomada de decisões (DO AMARAL MELLO, 2013).

A construção deste empreendimento foi ainda responsável por um processo de desterritorialização e reterritorialização na região de seu entorno (Altamira e outros municípios próximos), alterando a ocupação de parte deste território e gerando uma série de conflitos na população local afetada (urbana, rural, indígena e não indígena) (CORNÉLIO; NEVES; AMARAL, 2020). Principalmente na fase de implementação da usina, houve também um número significativo de trabalhadores, de outras regiões, atraídos para o local da obra em busca de emprego, novas oportunidades de negócios e desenvolvimento econômico, o que contribuiu para a modificação do espaço e da dinâmica populacional das áreas de influência do empreendimento (DE SOUZA et al., 2018).

Para De Santana e Brzezinsk (2018) existe uma grande disparidade entre as definições técnicas, teóricas e os desdobramentos práticos, no que tange os padrões de referência para os impactos ambientais deste tipo de empreendimento.

Um parâmetro que pode ser de grande utilidade nos estudos de dinâmica de urbanização é a Temperatura da Superfície Terrestre (TST) (BEZERRA et al. 2018). A TST fornece informações importantes sobre propriedades físicas do clima local, sendo esta, muito importante em processos ambientais, incluindo vegetação e escoamentos (BEZERRA et al., 2014; SANTANA, 2014). A utilização de geotecnologias pode auxiliar na detecção de mudanças no comportamento tanto de variáveis como a TST, através do fluxo de energia que chega e sai do alvo, quanto de índices espectrais, que combinam diferentes níveis de reflectância provenientes das imagens de satélites, fornecendo informações sobre áreas construídas, cobertura vegetal, solo e corpos hídricos (CAVALCANTE et al., 2020; ANA et al., 2019).

Um dos índices espectrais mais utilizados para identificar e avaliar a condição da vegetação num determinado local, bem como sua variação ao realizar a comparação entre dois períodos distintos, é o Normalized difference vegetation index (NDVI) (PAL; ZIAUL, 2017; BEZERRA et al., 2018). Outro índice que pode ser utilizado, neste caso para a identificação de áreas construídas, é o Normalized Difference Built-up Index (NDBI), importante na avaliação da variação de áreas urbanas (PAL; ZIAUL, 2017; GUHA et al., 2018).

Embora o emprego de técnicas de sensoriamento remoto e geoprocessamento sejam comuns em estudos e pesquisas ambientais, sobretudo como ferramenta importante para gestão de territórios e tomadas de decisões, as publicações nacionais relacionadas a este seguimento não costumam seguir um padrão metodológico bem descritivo, oportuno a replicações, diferentemente do modelo comumente adotado em pesquisas publicadas em periódicos internacionais 
(RAJESHWARI; MANI, 2014; GIANNINI et al., 2015; AVDAN; JOVANOVSKA, 2016; PAL; ZIAUL, 2017; ALEXANDER, 2020).

Neste contexto, objetivou-se com este trabalho aplicar técnicas de sensoriamento remoto na avaliação da influência da construção da Usina Hidrelétrica Belo Monte, em aspectos ambientais de mudança de uso e ocupação do solo e microclima, na região do entorno da usina. Foram adotadas ferramentas de aferição da TST, e os índices espectrais NDVI e NDBI. Para tanto, o período definido para o estudo considera o cenário anterior (ano de 2011), e posterior (ano de 2020) à consolidação do empreendimento.

\section{MATERIAL E MÉTODOS}

A Usina Hidrelétrica de Belo Monte (UHBM) está localizada no Rio Xingu, município de Vitória do Xingu, estado do Pará, próximo à confluência com a rodovia Transamazônica/BR230 (Figura 1). O processo de implementação da usina envolveu uma área total de inundação superior a $500 \mathrm{~km}^{2}$, sendo aproximadamente $380 \mathrm{~km}^{2}$ localizados no chamado Reservatório do Xingu (Rio Xingu) (ELETROBRAS, 2009).

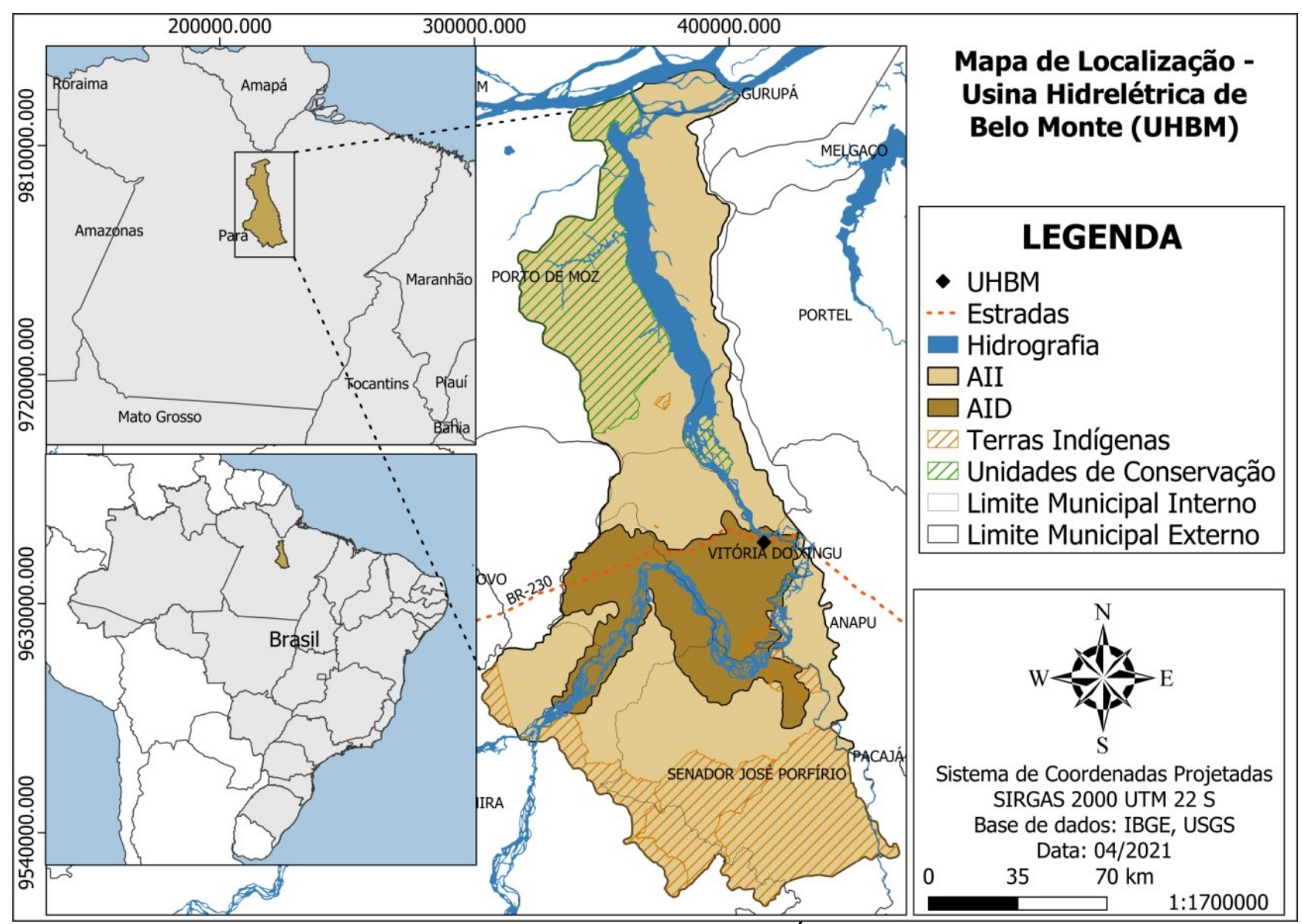

Figura 1 - Mapa de Localização da Área de Estudo Fonte: Os autores 
A área de estudo engloba parte de alguns municípios (Altamira, Anapu, Brasil Novo, Gurupá, Porto de Moz, Senador José Porfírio e Vitória do Xingu), que compõem a Área Diretamente Afetada (ADA) e as Áreas de Influência Direta (AID) e indireta (AII) da UHEBM (Figura 1). A AID é delimitada pela extensão dos impactos ocasionados pela instalação e operação do empreendimento, ocupando, ao todo, aproximadamente $4.600 \mathrm{~km}^{2}$. A ADA é ocupada por toda a infraestrutura necessária para a instalação e operação da Usina, incluindo também as estradas de acesso e as áreas inundadas, correspondendo a aproximadamente $1.500 \mathrm{~km}^{2}$. Já a AII - que sofre impactos indiretos decorrentes das alterações que aconteceram em áreas mais próximas - ocupa um espaço de aproximadamente $25.400 \mathrm{~km}^{2}$ (ELETROBRAS, 2009).

Os municípios em questão possuem, combinados, uma população de aproximadamente 250 mil habitantes. Os Índices de Desenvolvimento Humano Municipal (IDHM) estão considerados entre médio e baixo, variando de 0,503, no município de Porto de Moz, a 0,665, para Altamira (IBGE, 2021). Estão localizadas na região, diferentes unidades de conservação, como Reservas Extrativistas e Indígenas; Reserva de Desenvolvimento Sustentável; Refúgio da Vida Silvestre; Estação Ecológica; e Floresta Nacional.

O clima da região, segundo a classificação de Köppen, é caracterizado como tropical úmido (Am), apresentando uma temperatura média anual de $25^{\circ} \mathrm{C}$, com precipitação média de $2.300 \mathrm{~mm}$ anuais, sendo de janeiro a abril, o período de maior precipitação e, de julho a outubro, o de menor precipitação (INMET, 2021). A vegetação encontrada na área é caracterizada, principalmente, como floresta de terra firme (MPEG, 2002).

As variáveis ambientais avaliadas foram Temperatura de Superfície Terrestre (TST), NDVI e NDBI. Para tanto, procedeu-se, inicialmente, à aquisição de dados primários. Os procedimentos metodológicos envolvidos nesta fase do estudo seguiram as etapas dispostas na Figura 2.

Foram utilizadas imagens dos satélites LANDSAT 5 (sensor TM) e LANDSAT 8 (sensor OLI - Operational Land Imager), disponíveis na base de dados do United States Geological Survey (USGS). As imagens foram selecionadas para os meses de julho e início de agosto dos anos de 2011, anterior à construção da UHE de Belo Monte, e de 2020, após a consolidação do empreendimento (Tabela 1). As datas escolhidas correspondem ao período menos chuvoso na região amazônica, para uma melhor compreensão dos efeitos de supressão vegetal e aferição de temperaturas, devido à maior estabilidade atmosférica (ausência de nuvens).

Anterior ao processamento dos dados foi realizada a correção atmosférica e radiométrica das cenas a partir da conversão dos Números Digitais (ND) para radiância das bandas termais, e para reflectância das bandas "RED", "NIR" e "SWIR-1". As equações utilizadas para as correções das cenas são disponibilizadas pelo USGS. 
Figura 2 - Fluxograma das Etapas Realizadas na Metodologia da Pesquisa

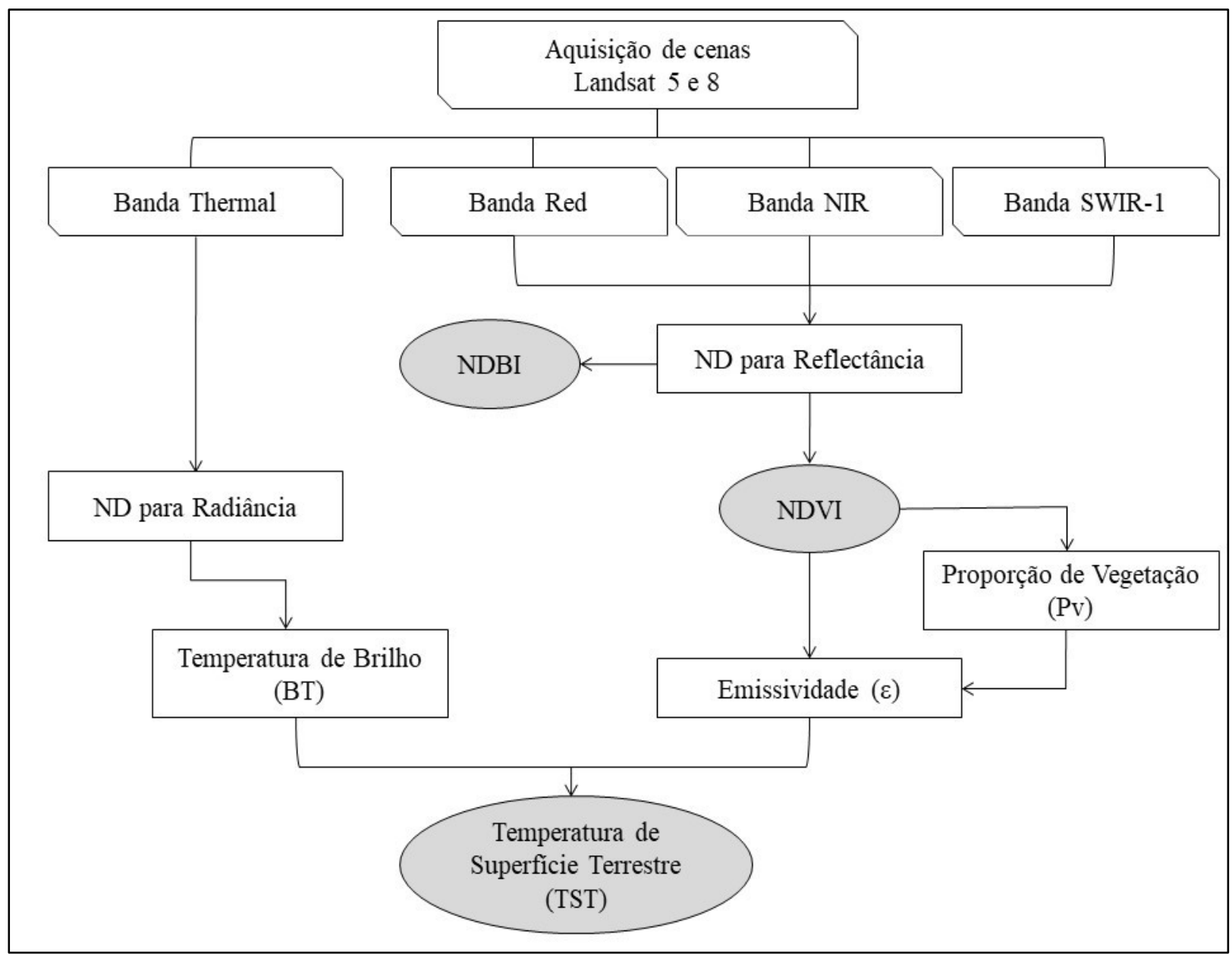

Fonte: Elaborado pelos autores.

Tabela 1 - Identificação das Imagens Orbitais Utilizadas no Estudo

\begin{tabular}{lcc}
\hline Satélite/Sensor & Data & Órbita/Ponto \\
\hline & $04 / 07 / 2011$ & $225 / 62$ \\
\cline { 2 - 3 } & & $225 / 63$ \\
\cline { 2 - 3 } LANDSAT 5 & & $226 / 61$ \\
\cline { 2 - 3 } & & $226 / 62$ \\
\hline & & $226 / 63$ \\
\hline & & $225 / 62$ \\
\cline { 2 - 3 } LANDSAT 8 & $04 / 08 / 2020$ & $226 / 61$ \\
\cline { 2 - 3 } & & $226 / 62$ \\
\cline { 2 - 3 } & & $226 / 63$ \\
\hline
\end{tabular}

Fonte: USGS, 2013 
Os índices NDVI e NDBI foram calculados a partir das equações 1 e 2.

$$
\begin{aligned}
& N D V I=\frac{(N I R-R E D)}{(N I R+R E D)} \\
& N D B I=\frac{S W I R-N I R}{S W I R+N I R}
\end{aligned}
$$

Em que, "RED" representa a banda do vermelho da faixa espectral; "NIR" é a banda correspondente à reflectância na região espectral do infravermelho próximo; e "SWIR" é a banda que corresponde à reflectância do infravermelho de onda curta.

O NDVI possibilita avaliar a atividade fotossintética da vegetação por meio do comprimento de onda da região do infravermelho próximo, e do vermelho. Os valores deste índice variam de -1 a 1 , em que os valores próximos a 0 (zero) representam uma superfície com baixa quantidade de vegetação ou não vegetada, enquanto que os próximos a 1 indicam um maior vigor vegetativo, já os valores negativos indicam hidrografia (DA SILVA LOTUFO et al., 2020). Já o NDBI, possibilita caracterizar áreas urbanas com base nas respostas espectrais das áreas construídas entre bandas do infravermelho próximo e infravermelho médio (PINHEIRO; LARANJEIRA, 2013).

Neste trabalho, considerou-se valores positivos de NDBI como representativos de áreas construídas, ao passo que os valores negativos representaram áreas em condições naturais (diferentes níveis de vegetação e hidrografia) (Tabela 2).

Tabela 2 - Classes Correspondentes aos Intervalos de NDVI e NDBI

\begin{tabular}{cc}
\hline Classe & NDVI \\
\hline Vegetação Densa & $1,0-0,6$ \\
Vegetação Secundária & $0,6-0,4$ \\
Vegetação Pobre & $0,4-0,2$ \\
Solo Exposto & $0,2-0,0$ \\
Hidrografia & $<0,0$ \\
\hline Classe & NDBI \\
\hline Vegetação e Hidrografia & $-1,0-0,0$ \\
Área Construída & $>0,0$ \\
\hline \multicolumn{2}{c}{ Fonte: Autores, 2021 }
\end{tabular}

Os dados de Temperatura de Brilho (BT), Proporção de Vegetação ( $\left.\mathrm{P}_{\mathrm{V}}\right)$ e Emissividade $(\varepsilon)$, necessários para a obtenção da TST, foram calculados a partir das equações 3, 4 e 5, respectivamente.

$$
B T=\left[\frac{K_{2}}{\operatorname{Ln}\left(\frac{K_{1}}{L_{\lambda}}+1\right)}\right]-273,15
$$


Onde, $B T$ é a temperatura de brilho; $K_{1}$ é a constante de calibração 1 (607,76 para Landsat 5 e 774,88 para Landsat 8); $K_{2}$ é a constante de calibração 2 (1260,56 para Landsat 5 e 1321,07 para Landsat 8); e $L_{\lambda}$ é a banda termal convertida para valores de radiância.

$$
P_{V}=\left(\frac{N D V I-N D V I_{S}}{N D V I_{V}-N D V I_{S}}\right)^{2}
$$

Onde, $P_{V}$ é a proporção de vegetação; $N D V I$ é o valor de NDVI calculado para a área de estudo; $N D V I_{S}$ é o valor de NDVI correspondente ao solo $(0,2)$; e $N D V I_{V}$ é o valor de NDVI correspondente à vegetação $(0,6)$.

$$
\varepsilon_{\lambda}=\varepsilon_{v \lambda} P_{v}+\varepsilon_{s \lambda}\left(1-P_{v}\right)
$$

Onde, $\varepsilon_{\lambda}$ é a emissividade; $\varepsilon_{v \lambda}$ corresponde à emissividade de vegetação $(0,973) ; \varepsilon_{s \lambda}$ corresponde à emissividade de solo exposto $(0,996)$; e $P_{v}$ é a proporção de vegetação (obtida na equação 4).

Procedeu-se a estimativa da TST de acordo com a metodologia de Avdan e Jovanovska (2016), por meio da equação 6 descrita por estes autores.

$$
T S T=\frac{B T}{\left\{1+\left[\left(\frac{\lambda B T}{\rho}\right) \operatorname{Ln} \varepsilon_{\lambda}\right]\right\}}
$$

Onde, TST é a temperatura de superfície terrestre; $B T$ é a temperatura de brilho obtida na equação $3 ; \lambda$ é o valor de radiância mais frequente da banda termal; $\rho$ corresponde ao valor de $1,1438 \times 10^{-2}$; e $\varepsilon_{\lambda}$ é a emissividade obtida na equação 5 .

Ainda para a análise de TST, foi utilizada somente a cena 225/62 dos anos 2011 e 2020, a qual contempla o espaço físico da UHE, além de partes da AID e AII (Figura 3). A opção de utilização de uma única cena foi motivada pela ausência de outras cenas captadas no mesmo dia pelo sensor escolhido; ao passo de a temperatura ser um parâmetro sensível a mínimos estímulos atmosféricos e suscetível a variações significativas em dias diferentes - condição limitante para uma análise mais completa e representativa da TST para toda a área de estudo.

Também foi realizada uma análise estatística de correlação de Pearson entre os índices NDVI x TST e NDBI x TST. Para tanto, foram coletados 125 pontos regulares amostrais, sobrepostos no recorte da cena 225/62, do ano de 2020, excluindo-se pontos sob a hidrografia (Figura 3). 
Figura 3 - Disposição dos Pontos Utilizados para Extração de Informações Aplicadas na Correlação Estatística de Variáveis Ambientais na Área Estudada

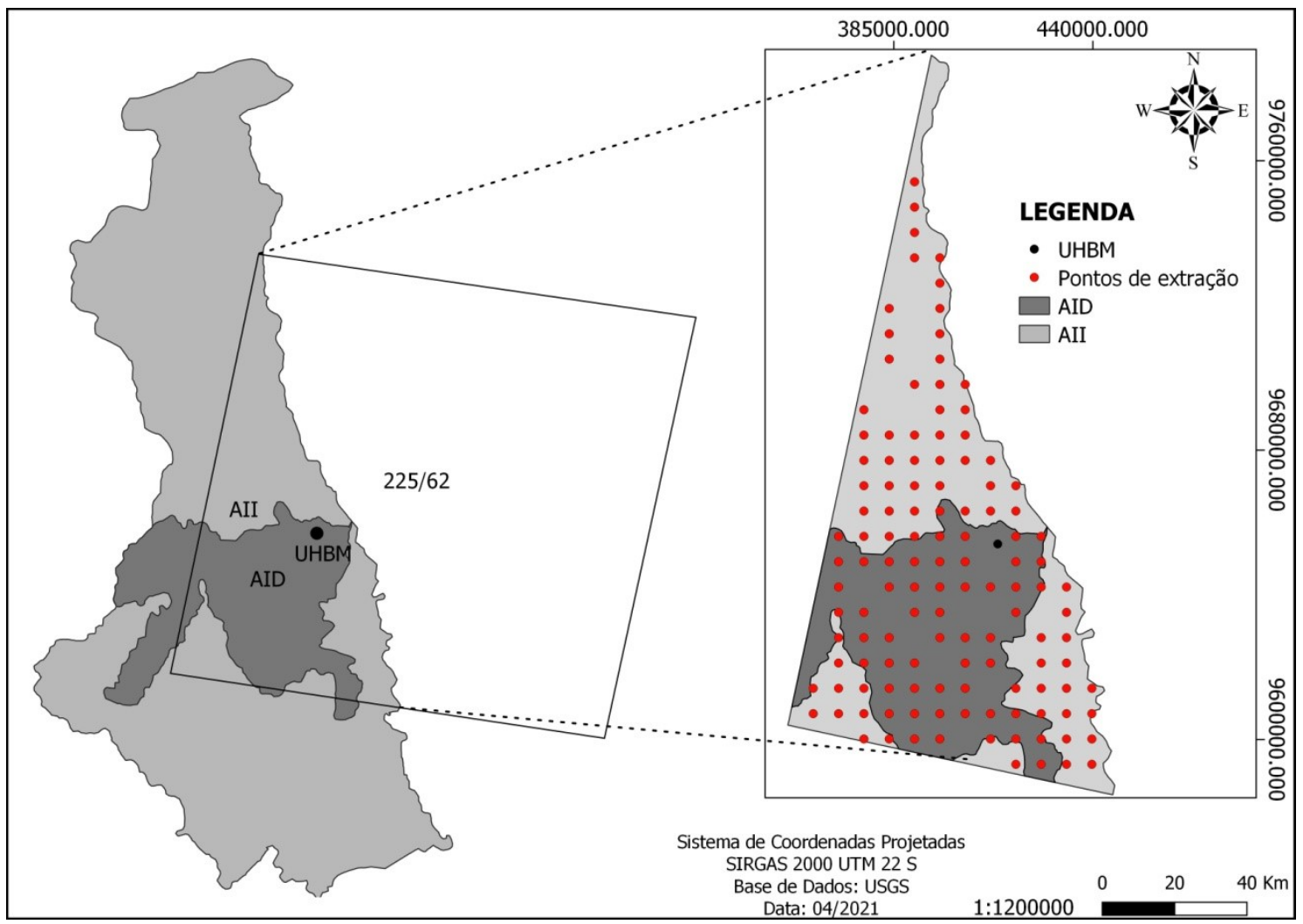

Fonte: Elaborado pelos autores.

Mediante a obtenção dos valores de TST, NDVI e NDBI, a partir das imagens de satélite, os dados gerados foram espacializados no programa QGis 3.16, para a geração de mapas representativos.

\section{RESULTADOS E DISCUSSÃO}

Observou-se um incremento da hidrografia na porção leste da área estudada no ano de 2020, em relação a 2011, sinalizada pela coloração vermelha e por valores negativos de NDVI (Figura 4). Isso reflete a área alagada pela construção da UHBM, a qual se encontra próximo à Volta Grande do Xingu.

Em 2011 a hidrografia representava 8,07\% da área avaliada, refletida em 2.017,86 km², saltando para 2.113,84 km² em 2020 (Tabela 3), o que indica aproximadamente $96 \mathrm{~km}^{2}$ de área alagada pela usina.

Houve uma redução de $1 \%$ de área para as classes "vegetação densa" e "vegetação secundária", entre 2011 e 2020 (Tabela 3). Já nas classes "vegetação pobre", "solo exposto" e "hidrografia", observaram-se aumentos de $0,57 \%, 0,55 \%$ e $0,37 \%$, respectivamente. 
Figura 4 - Mapa de NDVI dos anos de 2011 e 2020 para a Área de Influência Direta e Indireta da UHBM

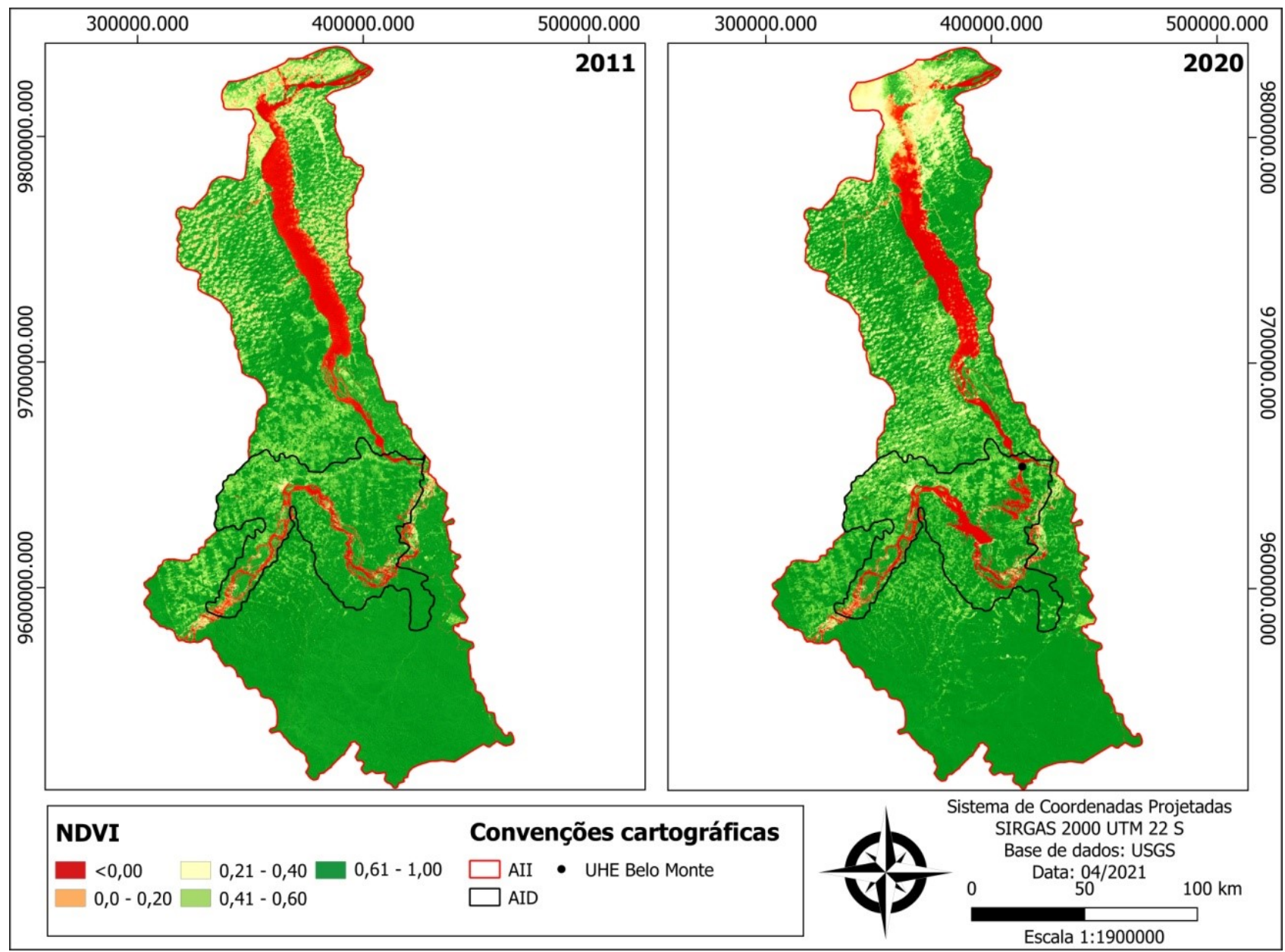

Fonte: Elaborado pelos autores.

Tabela 3 - Área Calculada para os anos de 2011 e 2020 em relação ao NDVI

\begin{tabular}{ccccc}
\hline \multirow{2}{*}{ Classes - NDVI } & \multicolumn{2}{c}{2011} & \multicolumn{2}{c}{2020} \\
\cline { 2 - 5 } & Área $\left(\mathbf{K m}^{\mathbf{2}}\right)$ & Área $\mathbf{( \% )}$ & Área $\left(\mathbf{K m}^{2}\right)$ & Área (\%) \\
\hline Vegetação Densa & $18.798,85$ & 75,21 & $18.685,67$ & 74,63 \\
Vegetação Secundária & $2.693,35$ & 10,78 & $2.471,24$ & 9,87 \\
Vegetação Pobre & 840,21 & 3,36 & 984,15 & 3,93 \\
Solo Exposto & 644,37 & 2,58 & 782,76 & 3,13 \\
Hidrografia & $2.017,86$ & 8,07 & $2.113,84$ & 8,44 \\
\hline
\end{tabular}

Fonte: Dados da pesquisa.

A diminuição referente às classes de vegetação está associada, além da construção da UHBM, à conversão em áreas de agropecuária (FAPESPA, 2020). Este comportamento pode ser observado no acréscimo de áreas em amarelo no padrão "espinha de peixe”, as quais correspondem ao intervalo de 0,21 a 0,40 de NDVI, refletidas em áreas de agropecuária nas porções sul e leste da AII (Figura 4) (SILVA JÚNIOR et al., 2014).

Os dados presentes no anuário estatístico do Pará de 2020 corroboram com o aumento das áreas destinadas à agricultura. De acordo com o anuário, os três municípios que compõem as 
maiores partes da AII e AID (Porto de Moz, Senador José Porfírio e Vitória do Xingú), juntos, apresentaram 4.872 hectares de área destinada à agricultura em 2011, saltando para 7.206 hectares em 2018. Os dados referentes ao ano de 2020 ainda não se encontram disponíveis no anuário (FAPESPA, 2020).

Os valores de NDVI apresentados para o período anterior ao início das obras da UHE (2011) variam de -0,95 a 0,82, com predominância de uma vegetação consolidada (Figura 4). Após a construção do empreendimento, a variação do NDVI foi de -0,81 a 0,87. Estima-se cerca de 300 $\mathrm{km}^{2}$ de área de vegetação moderada e consolidada, convertidas em solo exposto e ambiente alagado, em virtude do barramento de parte do Rio Xingu (Tabela 3).

O desmatamento e a supressão vegetal são consequências comuns da construção de Usinas Hidrelétricas, sejam de maior ou menor produção energética, causando prejuízos à fauna e flora local. A alteração na paisagem e inundações de áreas verdes também estão sempre presentes (LIMA; PINTO, 2017). A área alagada pela UHEBM impacta significativamente a Volta Grande do Xingu, um ecossistema singular e extensivamente estudado, berço de diversas espécies endêmicas de peixes, anfíbios, répteis e plantas, as quais são diretamente afetadas pela mudança de vazão (SAWAKUCHI et al., 2015; HERRERA; SANTANA, 2016; ZUANON et al., 2021).

De acordo com Zuanon et al. (2021), o desvio feito pelo canal de derivação da usina provocou uma diminuição de $80 \%$ da vazão da Volta Grande, em comparação a suas condições naturais. Ressalta-se ainda, que essa vazão residual é controlada pela concessionária da barragem e provoca alterações também na profundidade da água nesse trecho- processo intensificado nos diferentes períodos de cheia e seca. Segundo Freitas et al. (2015) a variação natural na profundidade das águas do rio Xingu é determinante na reprodução dos peixes, afetando o período reprodutivo, e consequentemente, as comunidades tradicionais que dependem da pesca na região.

Em relação ao NDBI, é possível observar a sede municipal de Altamira na porção Oeste da área, destacada em vermelho (Figura 5). Além disso, observa-se uma maior quantidade e espacialização de pontos em vermelho no ano de 2020, refletida na construção da UHEBM e, também, na expansão da urbanização dos municípios que compõem a área.

A variação do índice NDBI em 2011 era de $-0,86$ a 0,20, passando para -0,88 a 0,29 em 2020 (Figura 5). Os resultados sugerem que a construção da UHEBM motivou uma alteração no padrão estrutural da região, correspondendo a uma mudança do uso e ocupação do ambiente. 
Figura 5 - Mapa de NDBI dos anos de 2011 e 2020 para a Área de Influência Direta e Indireta da UHBM



Fonte: Elaborado pelos autores.

A área construída, que em 2011 correspondia a 0,69\% da área total avaliada, passou para 1,49\%, em 2020. Isso corresponde a aproximadamente $203 \mathrm{~km}^{2}$ ocupados por construções, antes não existentes no local, e representadas pelas estruturas físicas do empreendimento e ocupações residenciais no entorno (Tabela 4). Esses dados ilustram um processo de expansão urbana, iniciado em paralelo às obras da usina, ao passo que corroboram com resultados de outros autores (ALVES et al., 2018; CORNÉLIO; NEVES; AMARAL, 2020; FREIRE et al., 2018).

Tabela 4 - Área Calculada para os anos de 2011 e 2020 em relação ao NDBI

\begin{tabular}{ccccc}
\hline \multirow{2}{*}{ Classes - NDBI } & \multicolumn{2}{c}{$\mathbf{2 0 1 1}$} & \multicolumn{2}{c}{$\mathbf{2 0 2 0}$} \\
\cline { 2 - 5 } & Área $\left(\mathbf{K m}^{\mathbf{2}}\right)$ & Área (\%) & Área $\left(\mathbf{K m}^{\mathbf{2}}\right)$ & Área (\%) \\
\hline Vegetação e Hidrografia & $25.198,61$ & 99,3 & $25.004,76$ & 98,5 \\
Área Construída & 174,47 & 0,69 & 378,18 & 1,49 \\
\hline
\end{tabular}

Fonte: Dados da pesquisa.

Um dado que indica o desenvolvimento urbano na AII da UHE Belo Monte é o crescimento populacional nos municípios constituintes da maior parte da área. De acordo com o anuário estatístico do Pará, os municípios de Porto de Moz, Senador José Porfírio e Vitória do 
Xingú apresentaram, em conjunto, uma população de 61.202 habitantes em 2011, progredindo para 68.560 habitantes em 2020 (FAPESPA, 2020).

Outro dado que corrobora com o comportamento observado de expansão da área construída é o aumento das unidades consumidoras nos municípios citados anteriormente. Ainda de acordo com o anuário estatístico do Pará, as unidades consumidoras dos principais municípios constituintes da AII, agrupados, somaram 7.925 unidades em 2011, variando entre residenciais, industriais e comércios. Já para o ano de $2019^{1}$, houve um salto para 19.573 unidades consumidoras na área (FAPESPA, 2020).

Em relação a TST, no ano de 2011 as áreas de maior temperatura ficaram concentradas majoritariamente na porção Centro-oeste da área de estudo (Figura 6), refletidas nas áreas de agropecuária e ocupações populacionais, atingindo valores de $25,7^{\circ} \mathrm{C}$. O Rio Xingu, destacado em amarelo, apresentou em média $23,81^{\circ} \mathrm{C}$ no mesmo ano (Figura 6).

Já em 2020, após a consolidação do empreendimento, observa-se uma maior espacialização de áreas com maiores temperaturas, destacadas em vermelho (Figura 6). Esse comportamento pode estar relacionado à expansão de áreas destinadas à agricultura e da urbanização, as quais absorvem maiores quantidades de radiação pela ausência ou pouca quantidade de vegetação (AMORIM, 2019; FAPESPA, 2020). Além disso, a área construída da UHBM atingiu valores que variaram entre $33^{\circ}$ e $34^{\circ} \mathrm{C}$.

De modo geral, observou-se em relação a TST uma significativa elevação entre os anos, com uma diferença de mais de $8^{\circ} \mathrm{C}$ entre as máximas, atingindo $34{ }^{\circ} \mathrm{C}$ após a consolidação do empreendimento (Figura 6). As áreas vegetadas da região apresentaram, em média, TST de $28,9^{\circ} \mathrm{C}$, enquanto que as áreas construídas $33,3^{\circ} \mathrm{C}$.

Esse comportamento pode estar relacionado à conversão da superfície por materiais impermeáveis, provocada pelo desenvolvimento urbano. Superfícies como metais, concretos e asfalto possuem a capacidade de absorver maiores quantidades de radiação, o que reflete no aumento da temperatura superficial (AMORIM, 2019).

Em pesquisa feita por Pal e Ziaul (2017) em English Bazar, cidade indiana, os autores puderam observar o aumento do intervalo da TST entre os anos 1991, 2010 e 2014, assim como observado na UHEBM, associando esse comportamento ao crescimento de áreas urbanas, intensificadas pela abertura de estradas, as quais provocam o aumento da TST no entorno de seu eixo. Além disso, o aumento da TST pode ser provocado diretamente pela redução da vegetação, a qual engloba áreas que contribuem com a manutenção da temperatura pela transpiração das árvores.

\footnotetext{
${ }^{1}$ Dados de unidades consumidoras do ano de 2020 ainda não foram disponibilizados pela entidade responsável (FAPESPA, 2020).
} 
Figura 6 - Mapa do Comportamento da Temperatura de Superfície Terrestre (TST) na área do entorno da UHE Belo Monte nos anos de 2011 e 2020

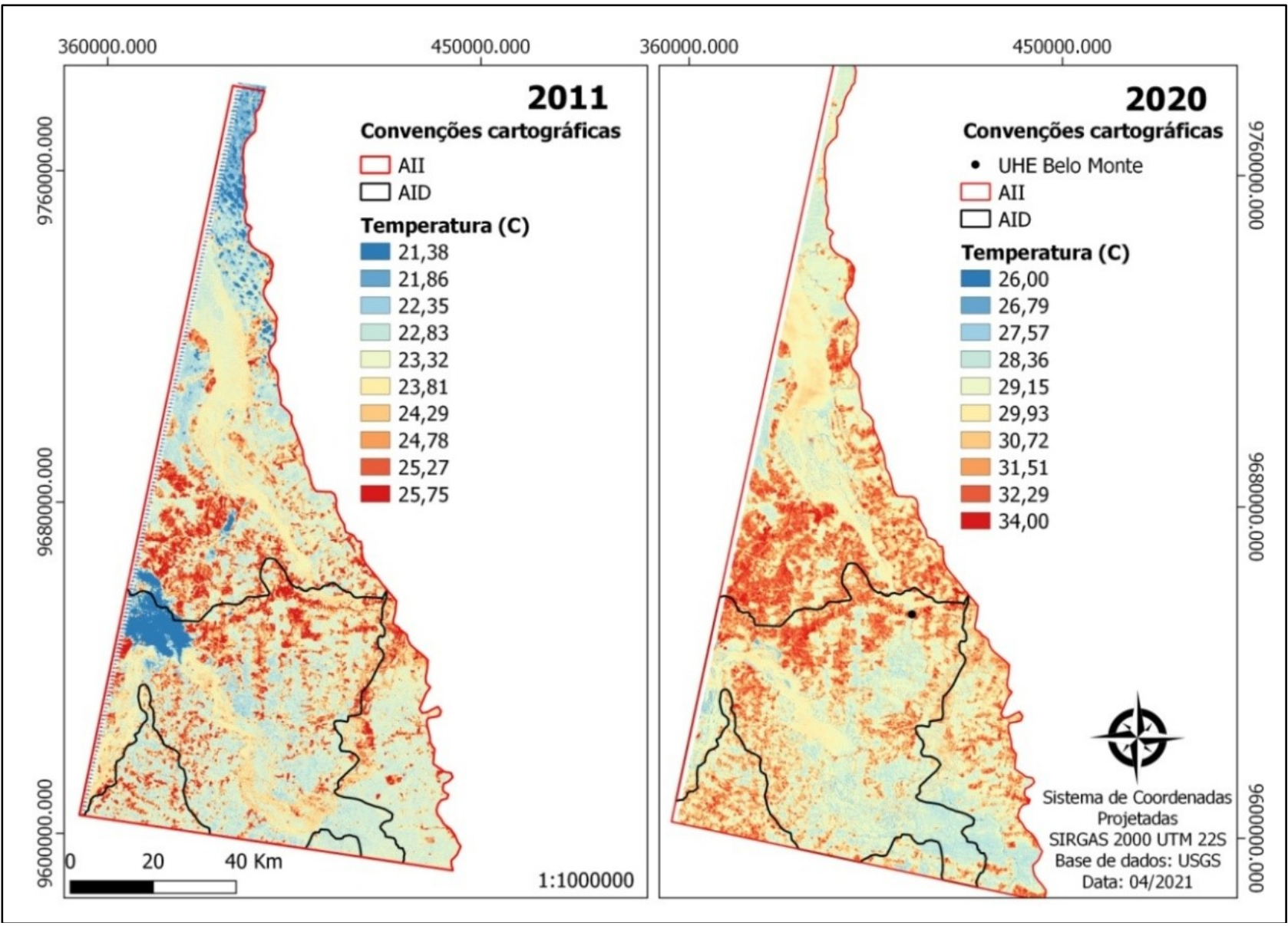

Fonte: Dados da pesquisa.

Os dados médios mensais de Temperatura do Ar (TA) da torre meteorológica da região mais próxima ${ }^{2}$ revelam ainda que, em comparação com os meses de julho de 2011 (TA média de $26,90^{\circ} \mathrm{C}$ ) e julho de 2020 (TA média de $28,4^{\circ} \mathrm{C}$ ), existiu uma alteração de menor expressão, se comparada às aferições do índice de Temperatura de Superfície na região do entorno da UHE. Isso pode ser atribuído a influência na temperatura exercida pelo empreendimento, com maior impacto nas AID e AII (ou parte destas).

Os resultados da correlação estatística entre os 125 pontos amostrais de controle demonstraram uma relação de influência negativa $(-0,48)$ e pouco significativa entre as variáveis TST e NDVI (Figura 7a). Assim, sugere-se que, em algum grau, os ambientes com ausência de vegetação ou com presença de vegetações menos expressivas, tendem a temperaturas mais elevadas. Quando comparados os índices TST e NDBI, observa-se uma correlação positiva $(0,69)$, de moderada significância (figura 7b), sugerindo de modo mais contundente, que ambientes construídos apresentam maiores temperaturas.

${ }^{2}$ Estação Meteorológica Convencional de Altamira, do Instituto Nacional de Meteorologia (INMET). 
Figura 7 - Correlação Estatística Linear de Pearson entre NDVI (A) e NDBI (B) com a Temperatura de Superfície

a) Temperatura $x$ NDVI

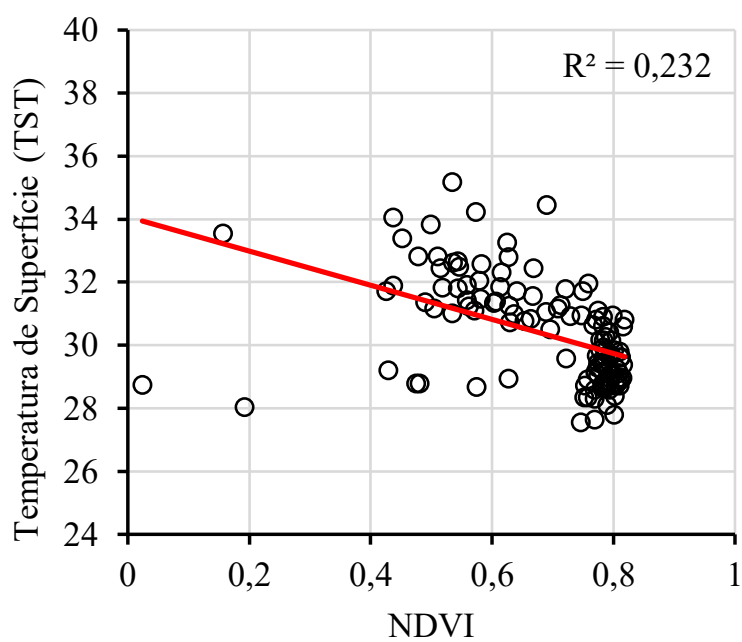

b) Temperatura $\mathrm{x}$ NDBI

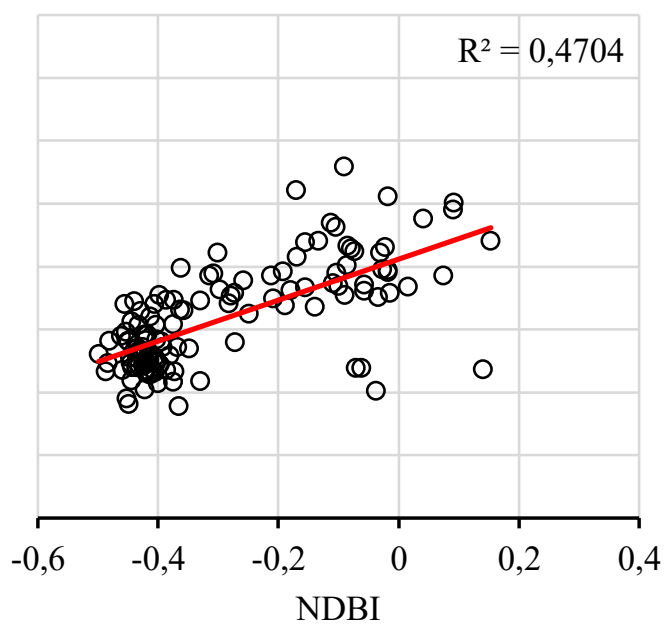

Fonte: Dados da pesquisa.

Em estudo similar para a região, Lobato et al. (2017) também estimaram um aumento da temperatura superficial, na ordem de $2^{\circ} \mathrm{C}$, após a consolidação deste mesmo empreendimento. Para estes autores, a nova ocupação urbana contribuiu decisivamente para esta alteração no microclima e sensação térmica local.

Em estudo realizado em reassentamentos urbanos coletivos em Altamira (processo de reterritorialização) sugere-se que, apesar de a maior parte dos indivíduos relatarem transformações positivas (principalmente pela nova estrutura ofertada), estes ainda observam expressivos impactos. Dentre eles, tem-se o exemplo da própria alteração no microclima local como uma limitação para a qualidade de vida (ALVES et al., 2018).

\section{CONCLUSÃO}

A construção da Usina Hidrelétrica Belo Monte gerou uma mudança expressiva no uso e ocupação da área construída e em seu entorno, com significativa alteração do microclima. Após a consolidação do megaempreendimento, a perda de parte da área vegetada e consequente conversão em ambiente construído ou alagado (aproximadamente $300 \mathrm{~km}^{2}$ ), sugere relação com o aumento das aferições de temperaturas de superfície máximas em até $8{ }^{\circ} \mathrm{C}$.

Ressalta-se, portanto, que as conclusões deste trabalho estão cerceadas a uma análise de representatividade moderada, devido às limitações no acesso aos dados. Sendo assim, objetivando resultados mais concretos, recomenda-se a utilização de um número maior de cenas (imagens de satélite), cobrindo período e perímetro maiores do que os adotados neste estudo, sobretudo para as aferições da Temperatura de Superfície da Terra. 
De modo geral, as técnicas de sensoriamento remoto utilizadas demonstraram boa resposta e baixa complexidade metodológica, apesar da limitação no acesso a dados indispensáveis. Logo, se inserem como ferramentas de grande utilidade para estudos técnicos, sendo válidas para a gestão ambiental e para a tomada de decisões.

\section{REFERÊNCIAS}

ALEXANDER, C. Normalized difference spectral indices and urban land cover as indicators of land surface temperature (LST). International Journal of Applied Earth Observation and Geoinformation, v. 86, p. 102013, 2020.

ALVES, L. S.; HAGE, S. N. D. S.; PEREIRA JÚNIOR, A. A Usina Hidrelétrica de Belo Monte (Altamira, Estado do Pará, Norte do Brasil), o reassentamento urbano coletivo e a avaliação de impactos ambientais. Revista Brasileira de Gestão Ambiental e Sustentabilidade, v. 5, n. 9, p. 49-74, 2018.

AMORIM, M. C. C. T. Ilhas de calor urbanas: métodos e técnicas de análise. Revista Brasileira de Climatologia, ano 15, Edição especial, XIII Simpósio Brasileiro de Climatologia Geográfica, jun. 2019.

ANA, C. B. S. O.; CANDEIAS, L. B.; JÚNIOR, J. R. T. Utilização de índices físicos a partir de imagens OLI-TIRS para o mapeamento de uso e cobertura da terra no entorno do aeroporto internacional do Recife/Guararapes-Gilberto Freire. Revista Brasileira de Geografia Física, v. 12, n. 03, p. 1039-1053, 2019.

ARAUJO, M. M. V.; DE JESUS PINTO, K.; DE OLIVEIRA MENDES, F. A Usina de Belo Monte e os impactos nas terras indígenas. Planeta Amazônia: Revista Internacional de Direito Ambiental e Políticas Públicas, n. 6, p. 43-51, 2015.

AVDAN, U.; JOVANOVSKA, G. Algorithm for automated mapping of land surface temperature using LANDSAT 8 satellite data. Journalofsensors, v. 2016, 8 p. 2016.

BEZERRA, J. M.; MOURA, G. B. D. A.; SIlvA, B. B. D.; LOPES, P. M.; SILVA, E. F. D. F. Parâmetros biofísicos obtidos por sensoriamento remoto em região semiárida do estado do Rio Grande do Norte, Brasil. Revista Brasileira de Engenharia Agrícola e Ambiental, v. 18, n. 1, p. 73-84, 2014.

BEZERRA, P. E. S.; DE MORAES, E. T. I.; DA COSTA SOARES, I. R. Análise da Temperatura de Superfície e do Índice de Vegetação no Município de Belém na Identificação das Ilhas de Calor. Revista Brasileira de Cartografia, v. 70, n. 3, p. 803-818, 2018.

CARRARO, F. G. P. Análise da cobertura do solo usando NDVI, na região da hidrelétrica de Belo Monte, estado do Pará - Brasil. Orientador: Heleno Fulber. 2019. 109 f. Dissertação (Mestrado em Engenharia de Barragem e Gestão Ambiental) - Núcleo de Desenvolvimento Amazônico em Engenharia, Universidade Federal do Pará, Tucuruí, 2019.

CAVAlCANTE, J. C.; AlMEIDA, C. A.; CASTElO, T. B. Análise De NDVI E Temperatura Da Superfície Terrestre Utilizando A Plataforma Do Google Earth Engine Em Área De Savana Amazônica E Floresta Secundária, No Município De Vigia - PA. Revista de Geociências do Nordeste, v. 6, n. 1, p. 64-70, 29 jun. 2020. 
COELHO, A. L. N.; CORREA, W. S. C. Temperatura de Superfície Celsius do Sensor TIRS/Landsat-8: metodologia e aplicações. Revista Geográfica Acadêmica, v. 7, n. 1, p. 31-45, 2013.

CORNÉliO, G. S.; NEVES, I. C.; AMARAL, M. D. B. Usina De Belo Monte E O Processo De Des-Re-Territorialização Dos Atingidos Urbanos Em Altamira/PA. InterEspaço: Revista de Geografia e Interdisciplinaridade, v. 5, n. 18, p. 15837, 2020.

DA SILVA LOTUFO, J. B.; MACHADO, N. G.; DE MELLO TAQUES, L.; DE SOUZA, D. M.; MÜTZENBERG, N. L. N.; BIUDES, M. S. Índices Espectrais e Temperatura de Superfície em Áreas Queimadas no Parque Estadual do Araguaia em Mato Grosso. Revista Brasileira de Geografia Física, v. 13, n. 02, p. 648-663, 2020.

DE SANTANA, A. T.; BRZEZINSKI, M. L. N. L. UHE Belo Monte: o estudo de impacto ambiental e suas contradições. Revista Brasileira de Iniciação Científica, v. 5, n. 2, p. 235-256, 2018.

DE SOUZA, D. P.; DA SILVA, W. R. S.; CERVINSKI, G. C.; DOS SANTOS, B. D.; DE ASSIS COMARÚ, F.; TRIGOSO, F. B. M. Desenvolvimento urbano e saúde pública: impactos da construção da UHE de Belo Monte. Desenvolvimento e Meio Ambiente, v. 46, 2018.

DO AMARAL MELLO, C. C. Se houvesse equidade: a percepção dos grupos indígenas e ribeirinhos da região da Altamira sobre o projeto da Usina Hidrelétrica de Belo Monte. Novos Cadernos NAEA, v. 16, n. 1, 2013.

ELETROBRAS - Centrais Elétricas Brasileiras. Aproveitamento Hidrelétrico Belo Monte. Brasília, 100 p. Maio 2009. Disponível em: <https://eletrobras.com/pt/AreasdeAtuacao/geracao/ belo_monte/Rima-AHE-Belo-Monte.pdf>

FAPESPA - FUNDAÇÃO AMAZÔNIA DE AMPARO A ESTUDOS E PESQUISAS. Anuário Estatístico do Pará. Belém, 2020. Disponível em: $<\underline{\text { http://www.fapespa.pa.gov.br/menu/170 }>}$

FERREIRA, A. M. M.; SALATI, E. Forças de transformação do ecossistema amazônico. Estudos avançados, v. 19, n. 54, p. 25-44, 2005.

FREIRE, L. M.; DE LIMA, J. S.; DA SILVA, E. V. Belo Monte: fatos e impactos envolvidos na implantação da usina hidrelétrica na região Amazônica Paraense. Sociedade \& Natureza, v. 30, n. 3, p. 18-41, 2018.

FREITAS, T. M. S.; PRUDENTE, B. S.; OLIVEIRA, V. A.; OLIVEIRA, M. N. C.; PRATA, E. G.; LEÃO, H.; MONTAG, L. F. A. Influenceoftheflood pulse onthereproductionofTocantinsia piresi (Miranda Ribeiro) andAuchenipterus nuchalis (Spix \&Agassiz) (Auchenipteridae) ofthemiddle Xingu River, Brazil. Brazilian Journal of Biology, v. 75, n. 3, p. 158-167, 2015.

GIANNINI, M. B.; BELFIORE, O. R.; PARENTE, C.; SANTAMARIA, R. Land Surface Temperature from Landsat 5 TM images: comparison of different methods using airborne thermal data. Journal of Engineering Science \& Technology Review, v. 8, n. 3, 2015.

GUHA, S.; GOVIL, H.; DEY, A.; GILL, N. Analytical study of land surface temperature with NDVI and NDBI using Landsat 8 OLI and TIRS data in Florence and Naples city, Italy. EuropeanJournalof Remote Sensing, v. 51, n. 1, p. 667-678, 2018. 
HERRERA, J. A.; SANTANA, N. C. Empreendimento hidrelétrico e famílias ribeirinhas na Amazônia: desterritorialização e resistência à construção da hidrelétrica Belo Monte, na Volta Grande do Xingu. GEOUSP Espaço e Tempo (Online), v. 20, n. 2, p. 250-266, 2016.

IBGE - Instituto Brasileiro de Geografia e Estatística. Cidades - Panorama [online]. Índice de Desenvolvimento Humano Municipal. 2021. Disponível em: $<$ https://cidades.ibge.gov.br/brasil/pa/altamira/panorama $>$

IBGE - Instituto Brasileiro de Geografia e Estatística. Estimativas da População Residente Nos Municípios Brasileiros Com Data De Referência em $1^{\circ}$ de julho de 2020. IBGE: Rio de Janeiro, 2020. Disponível em: $<$ https://www.ibge.gov.br/estatisticas/sociais/populacao/9103-estimativas-depopulacao.html? $=\& \mathrm{t}=$ resultados $>$

INMET - Instituto Nacional de Meteorologia. Banco de Dados Meteorológicos para Ensino e Pesquisa, 2021.Disponível em: <https://bdmep.inmet.gov.br $>$

KOPPEN, W. Das geographische System der Klimate. - KOPPEN, W., R. GEIGER (Eds.): Handbuch der Klimatologie. - GebrüderBornträger, Berlin, 1, 1-44, part C. 1936.

LIMA, C. C.; PINTO, J. B. M. As audiências públicas e o diálogo dos saberes: uma abordagem sobre a instalação de hidrelétricas e seus impactos ambientais. Revista Brasileira de Direito, v. 13, n. 2, p. 137-154, 2017.

LOBATO, A. A. C.; GOVEIA, P. M. S.; DOS SANTOS, T. S.; DE PINHO ALVAREZ, W. A influência da bacia hidrográfica no clima urbano da Amazônia: análise do clima na bacia do Igarapé Ambé no perímetro urbano de Altamira-PA, no período de implantação da UHE Belo Monte, 2011 a 2015. Os Desafios da Geografia Física na Fronteira do Conhecimento, v. 1, p. 2691-2697, 2017.

MPEG - Museu Paraense Emílio Goeldi. Diagnóstico da vegetação e da flora da área de estudo da Usina Hidrelétrica de Belo Monte, Estado do Pará. Belém: Convênio MCTMPEG/ELETRONORTE. Relatório Técnico, 171 p. 2002.

NASCIMENTO, D. T. F.; DE OLIVEIRA, I. J. Análise Da Evolução Do Fenômeno De Ilhas De Calor No Município De Goiânia-GO (1986-2010). BoletimGoiano De Geografia, v. 31, n. 2, p. 113-127, 2011.

PAL, S.; ZIAUL, S. K. Detection of land use and land cover change and land surface temperature in English Bazar urban centre. The Egyptian Journal of Remote Sensing and Space Science, v. 20, n. 1, p. 125-145, 2017.

PINHEIRO, C.; LARANJEIRA, M. Análise do ambiente térmico e das condições de ventilação para a definição das funções climáticas na área urbana de Guimarães. Revista de Geografia e Ordenamento do Território, v. 1, n. 4, p. 249-272, 2013.

RAJESHWARI, A.; MANI, N. D. Estimation of land surface temperature of Dindigul district using Landsat 8 data. International Journal of Research in Engineering and Technology, v. 3, n. 5, p. 122-126, 2014.

SANTANA, N. C. Investigação de ilhas de calor em Brasília: análise multitemporal com enfoque na cobertura do solo. Revista Brasileira de Geografia Física, v. 7, n. 6, p. 1044-1054, 2014.

SAWAKUCHI, A. O.; HARTMANN G. A.; SAWAKUCHI, H. O.; PUPIM, F. N.; BERTASSOLI, D. J.; PARRA, M.; ANTINAO, J. L.; SOUSA, L. M.; SABAJ PÉREZ, M. H.; OLIVEIRA, P. E.; 
SANTOS, R. A.; SAVIAN, J. F.; GROHMANN, C. H.; MEDEIROS, V. B.; MCGLUE, M. M.; BICUDO, D. C.; FAUSTINO S. B. The Volta Grande do Xingu: reconstruction of past environments and forecasting of future scenarios of a unique Amazonian fluvial landscape. Scientific Drilling, v. 20, p. 21-32, 2015.

SILVA JÚNIOR, C. A.; NANNI, M. R.; DA SILVA, A. A.; CEZAR, E.; ROMAGNOLI, F. Conversion of land use and cover in northwest Amazon (Brazil). PesquisaAgropecuária Tropical, v. 44, n. 3, p. 230-237, 2014.

USGS - Geological Survey. Using the USGS Landsat Level-1 Data Product. 2013. Disponível em: $<$ https://www.usgs.gov/core-science-systems/nli/landsat/using-usgs-landsat-level-1-dataproduct>. Acesso em: 22 fev. 2021.

ZUANON, J.; SAWAKUCHI, A.; CAMARGO, M.; WAHNFRIED, I.; SOUSA, L.; AKAMA, A.; MURIEL-CUNHA, J.;RIBAS, C.; D'HORTA, F.; PEREIRA, T.; LOPES, P.; MANTOVANELLI, T.; LIMA, T. S.; GARZÓN, B.; CARNEIRO, C.; REIS, C. P.; ROCHA, G.; SANTOS, A. L.; DE PAULA, E. M.; PENNINO, M.; PEZZUTI, J. Condições para a manutenção da dinâmica sazonal de inundação, a conservação do ecossistema aquático e manutenção dos modos de vida dos povos da volta grande do Xingu. Papers do NAEA, v. 1, n. 2, 2021. 